\title{
Application of Wavelet Neural Network in Building Settlement Prediction
}

\author{
Ruijie Zhang ${ }^{1}$ \\ ${ }^{1} 845$ Rue Sherbrooke Ouest, Civil Engineering, McGill University, Montreal, Quebec, H3A 0G4, Canada
}

\begin{abstract}
Deformation monitoring, as a key link of information construction, runs through the entire process of the building design period, construction period and operation period[1]. At present, more mature static prediction methods include hyperbolic method, power polynomial method and Asaoka method. But these methods have many problems and shortcomings. In this paper, based on the characteristics of building foundation settlement and the methods widely discussed in this field, a wavelet neural network model with self-learning, self-organization and good nonlinear approximation ability is applied to the prediction problem of building settlement[2]. Using comparative analysis and induction method. The 20-phase monitoring data representing the deformation monitoring points of different settlement states of the line tunnel, using the observation data sequence of the first 15 phases respectively to take the cumulative settlement and interval settlement as training samples, through the BP artificial neural network and the improved wavelet neural network, for the last five periods Predict the observed settlement.Through the comparison, it is found that whether the interval settlement or the cumulative settlement is used, the prediction results of the wavelet neural network are basically better than the prediction results of the BP artificial neural network, and the number of trainings is greatly reduced. The adaptive prediction of the wavelet neural network. The ability is particularly obvious, and the prediction accuracy is significantly improved. Therefore, it can be shown that the wavelet neural network is indeed used in the settlement monitoring and forecast of buildings, which can obtain higher prediction accuracy and better prediction effect, and is a prediction method with great development potential.
\end{abstract}

\section{INTRODUCTION}

With the rapid development of the city, and in order to pursue more residential space, various high-rise buildings are rising from the ground, while in the buildings under load, the ground gene is disturbed by the load, causing the building to settle. The uniform settlement within a reasonable range may have little effect on the building, but if the building has uneven and serious settlement, the building's safe use will be affected and maintenance will even directly endanger the lives and property of the residents[3]. Although the settlement of the building itself has certain regularity, it is not necessarily uniform, so the settlement will affect the building itself. In order to avoid the occurrence of accidents, it is necessary to predict the settlement and deformation of buildings.

The prediction of building settlement is with many influencing factors extremely complex. The traditional static prediction method can only predict and calculate the final settlement, but cannot predict in real time. Therefore, for the purpose of real-time prediction, we can try to apply neural network to the problem of building settlement.

Artificial neural network has the characteristics of large-scale parallel processing, distributed storage, high redundancy and nonlinear calculation. Therefore, it has a very high computing speed, strong association ability, adaptability and self-organization ability. These characteristics are very suitable for real-time prediction of building settlement.

It can be seen from this that timely, accurate and scientific analysis of settlement deformation monitoring data and prediction of the settlement settlement of buildings have very important guiding significance for the construction and operation stages of construction projects.

In this paper, based on the characteristics of building foundation settlement, by selecting the monitoring data of a subway line tunnel, using cumulative settlement and interval settlement as training samples, through the BP artificial neural network and improved wavelet neural network, the last five phases of settlement prediction are predicted. It is concluded that the wavelet neural network can be used in building settlement monitoring and forecasting, which provides certain basis and guidance for building settlement monitoring.

\section{TRADITIONAL STATIC PREDICTION METHODS}


At present, there are many methods based on building settlement prediction, which can be mainly divided into two categories:

The first type is based on theoretical calculation methods, including finite element method, finite difference method, numerical analysis method, etc.[4]. It is based on the classic mechanics created by Taishaji et al.[4]. Besides, it introduces many simplified assumptions of traditional settlement prediction methods and empirical theories that are continuously developed, perfected, and improved, represented by theory[4]. Although the empirical method is simple and practical and is more realistic for solving problems in engineering in a timely manner, it only gives a rough calculation formula or a range. Many of them rely too much on indoor tests, and the test workload is quite large, and some even have additional conditions. The relevant parameters need to be retrieved from the respective statistical tables, and the deviation between the calculated result and the measured value is generally large. At the same time, due to the wide variety of foundations in various regions, the construction methods and construction conditions are very different. Meanwhile, various geological conditions are difficult to determine, so the theory-based calculation method is mainly used in building design work, and it is difficult to apply it to actual engineering prediction.

The other is the analysis method based on measured data. This method simulates and predicts the deformation value by establishing a data model, which mainly includes the regression analysis method, time series analysis model, gray system theory model, filtering model, artificial neural network model, etc. . In order to make up for the limitations of a single model and make better use of the advantages of various models, a combination of multiple models has been developed.

In short, the traditional static prediction method is only applicable to the case where the influencing factors are relatively simple. If the construction conditions and geological environment are complex, the application of this method will be greatly restricted, and the deviation of the predicted results from the actual results will also increase accordingly.

\section{WAVELET NEURAL NETWORKS}

\subsection{Wavelet neural network}

\subsubsection{Overview of wavelet analysis}

Wavelet transform is a kind of signal time-frequency analysis method. The main method is the expansion and contraction and Fourier transform only provides information about the frequency domain, so that the local information about the time is basically lost[5]. Unlike Fourier transform, wavelet transform is through the scaling of the width of the base wavelet (mother wavelet) to obtain the signal frequency characteristics, and then through the mobile base wavelet to obtain the signal of time information base wavelet scaling. In addition the translation is calculation of wavelet coefficients, which can be used to reflect the relevance of the wavelet and the local signal

\subsubsection{Combination of wavelet analysis and neural network}

Wavelet neural network is obtained by combining wavelet analysis and neural network. Neural networks rely on autonomous learning to achieve certain mapping functions. As long as the learning data is complete enough, no matter whether the system is nonlinear or not, it can successfully find a suitable mapping. The arbitrary approximation characteristics of nonlinear continuous functions in wavelet analysis are well distinguished in time and frequency, making the wavelet neural network constructed based on wavelet analysis theory more suitable for learning local nonlinear and rapidly changing functions. Therefore, on the basis of wavelet transform theory, combined with neural network function, wavelet neural network can be formed.

According to the characteristics of the combination of wavelet analysis theory and neural network, wavelet neural network is divided into two categories from the structural form:

(1) The auxiliary wavelet neural network is the combination of wavelet transform and neural network. Wavelet analysis, as a pre-processing method of neural network, provides input processing.

(2)The embedded wavelet neural network is the combination of wavelet decomposition and embedded fusion of neural network., wavelet and neural network are directly fused, and wavelet function and scale function form network neurons.

\subsubsection{Features of wavelet neural network}

The wavelet neural network that emerged as a simulation of the human brain has the following characteristics:

(1) Parallel structure and parallel processing. The calculation and storage of the wavelet neural network are related to each other in time and space,. They are parallel in time, and located in the same area in space.

(2) Distributed storage of information. In the wavelet neural network, a single wavelet base and the entire wavelet neural network have the dual functions of information processing and storage.Besides, these two functions are naturally integrated,with the storage of information reflected in the interconnection of the wavelet base in the distributed networks, which is called distributed storage.

(3) Fault tolerance. Wavelet neural network has the ability to make complete and correct conclusions based on incomplete noise information.

(4) Self-organization, self-learning and adaptive reasoning ability. The wavelet neural network has the self-organizing ability of extracting external input signal features by itself and can develop the self-learning ability of knowledge. Also it can correctly distinguish and process the adaptive reasoning ability of various different input modes of the network during training. 
(5) Non-linear. Since the wavelet functions that make up the wavelet neural network are non-linear, the wavelet neural network is a complex dynamic system with large-scale nonlinear combinations.

\subsection{Example of Settlement Prediction of Wavelet Neural Network}

\subsubsection{Selection of training samples}

In this paper, by selecting 20 monitoring data representing deformation monitoring points of different settlement

Table 1 Data sheet on settlement of settlement monitoring points (unit: $\mathrm{mm}$ )

\begin{tabular}{|c|c|c|c|c|c|c|c|c|c|c|}
\hline $\begin{array}{c}\text { Number of } \\
\text { observation } \\
\text { periods }\end{array}$ & $\begin{array}{c}\text { Period } \\
1\end{array}$ & $\begin{array}{c}\text { Period } \\
2\end{array}$ & $\begin{array}{c}\text { Period } \\
3\end{array}$ & $\begin{array}{c}\text { Period } \\
4\end{array}$ & $\begin{array}{c}\text { Period } \\
5\end{array}$ & $\begin{array}{c}\text { Period } \\
6\end{array}$ & $\begin{array}{c}\text { Period } \\
7\end{array}$ & $\begin{array}{c}\text { Period } \\
8\end{array}$ & $\begin{array}{c}\text { Period } \\
9\end{array}$ & $\begin{array}{c}\text { Period } \\
10\end{array}$ \\
\hline $\begin{array}{c}\text { Interval } \\
\text { settlement }\end{array}$ & 0.00 & 6.45 & 5.91 & 0.96 & 3.77 & 6.17 & -0.02 & 4.54 & 2.75 & 1.00 \\
\hline $\begin{array}{c}\text { Cumulative } \\
\text { settlement }\end{array}$ & 0.00 & 6.45 & 12.36 & 13.32 & 17.09 & 23.27 & 23.24 & 27.78 & 30.53 & 31.52 \\
\hline $\begin{array}{c}\text { Number of } \\
\text { observation } \\
\text { periods }\end{array}$ & $\begin{array}{c}\text { Period } \\
11\end{array}$ & $\begin{array}{c}\text { Period } \\
12\end{array}$ & $\begin{array}{c}\text { Period } \\
13\end{array}$ & $\begin{array}{c}\text { Period } \\
14\end{array}$ & $\begin{array}{c}\text { Period } \\
15\end{array}$ & $\begin{array}{c}\text { Period } \\
16\end{array}$ & $\begin{array}{c}\text { Period } \\
17\end{array}$ & $\begin{array}{c}\text { Period } \\
18\end{array}$ & $\begin{array}{c}\text { Period } \\
19\end{array}$ & $\begin{array}{c}\text { Period } \\
20\end{array}$ \\
\hline $\begin{array}{c}\text { Interval } \\
\text { settlement }\end{array}$ & 0.72 & 1.53 & 0.60 & 0.26 & 0.41 & 0.63 & 0.60 & 1.33 & 0.17 & 0.84 \\
\hline $\begin{array}{c}\text { Cumulative } \\
\text { settlement }\end{array}$ & 32.24 & 33.77 & 34.37 & 34.63 & 35.04 & 35.67 & 36.27 & 37.59 & 37.77 & 38.60 \\
\hline
\end{tabular}

\subsubsection{Comparison of prediction results}

Based on the above data, BP artificial neural network and wavelet neural network are used to make predictions. The prediction results are shown in Table 2 and 3 . It can be seen from the following table that the number of training states in a subway line tunnel[6], the cumulative settlement and interval settlement are used as training samples using the observation data sequence of the previous 15 periods, respectively, through BP artificial neural network and improved wavelet. The neural network predicts the amount of settlement observed in the last five phases.

The settlement observation data on the first 15 phases are divided into 8 training samples, with 8 values in each group. The first 7 values are used as the input of the network input node, while the last one is used as the expected value of the output node, as shown in Table 1 times of BP artificial neural network in interval settlement prediction is 8637 , and the number of training times in cumulative settlement prediction is 5864. In the interval settlement prediction, the number of training times of wavelet neural network is 231 , while in the cumulative settlement prediction, the number of training times is 223 .

Table 2 BP artificial neural network prediction results (unit: $\mathrm{mm}$ )

\begin{tabular}{|c|c|c|c|c|c|c|c|}
\hline \multirow{2}{*}{$\begin{array}{c}\text { Number of } \\
\text { observation } \\
\text { periods }\end{array}$} & \multirow{2}{*}{$\begin{array}{l}\text { Measured } \\
\text { value }\end{array}$} & \multicolumn{3}{|c|}{ Interval settlement prediction } & \multicolumn{3}{|c|}{ Cumulative settlement prediction } \\
\hline & & $\begin{array}{c}\text { Training } \\
\text { times }\end{array}$ & $\begin{array}{l}\text { Predictive } \\
\text { value }\end{array}$ & $\begin{array}{c}\text { Absolute } \\
\text { error }\end{array}$ & $\begin{array}{c}\text { Training } \\
\text { times }\end{array}$ & $\begin{array}{c}\text { Predictive } \\
\text { value }\end{array}$ & $\begin{array}{c}\text { Absolute } \\
\text { error }\end{array}$ \\
\hline Period 16 & 35.67 & \multirow{5}{*}{8637} & 34.80 & 0.87 & \multirow{5}{*}{5864} & 35.46 & 0.21 \\
\hline Period 17 & 36.27 & & 34.94 & 1.33 & & 35.74 & 0.53 \\
\hline Period 18 & 37.59 & & 35.05 & 2.54 & & 36.08 & 1.51 \\
\hline Period 19 & 37.77 & & 35.14 & 2.63 & & 36.53 & 1.24 \\
\hline Period 20 & 38.60 & & 35.23 & 3.37 & & 37.18 & 1.42 \\
\hline \multicolumn{2}{|c|}{ Model accuracy } & \multicolumn{3}{|c|}{2.33} & \multicolumn{3}{|c|}{1.11} \\
\hline
\end{tabular}

Table 3 The wavelet neural network prediction results (unit: $\mathrm{mm}$ )

\begin{tabular}{|c|c|c|c|c|c|c|c|}
\hline \multirow{2}{*}{$\begin{array}{c}\text { Number of } \\
\text { observation } \\
\text { periods }\end{array}$} & \multirow[b]{2}{*}{$\begin{array}{c}\text { Measured } \\
\text { value }\end{array}$} & \multicolumn{3}{|c|}{ Interval settlement prediction } & \multicolumn{3}{|c|}{ Cumulative settlement prediction } \\
\hline & & $\begin{array}{c}\text { Training } \\
\text { times }\end{array}$ & $\begin{array}{l}\text { Predictive } \\
\text { value }\end{array}$ & $\begin{array}{l}\text { Absolute } \\
\text { error }\end{array}$ & $\begin{array}{c}\text { Training } \\
\text { times }\end{array}$ & $\begin{array}{l}\text { Predictive } \\
\text { value }\end{array}$ & $\begin{array}{c}\text { Absolute } \\
\text { error }\end{array}$ \\
\hline Period 16 & 35.67 & \multirow{5}{*}{231} & 35.69 & 0.02 & \multirow{5}{*}{223} & 35.31 & 0.36 \\
\hline Period 17 & 36.27 & & 35.81 & 0.46 & & 36.30 & 0.03 \\
\hline Period 18 & 37.59 & & 36.01 & 1.58 & & 36.64 & 0.95 \\
\hline Period 19 & 37.77 & & 36.38 & 1.39 & & 37.55 & 0.22 \\
\hline Period 20 & 38.60 & & 36.53 & 2.07 & & 38.41 & 0.19 \\
\hline \multicolumn{2}{|c|}{ Model accuracy } & \multicolumn{3}{|c|}{1.41} & \multicolumn{3}{|c|}{0.75} \\
\hline
\end{tabular}

Through comparing the results predicted by these two methods with the actual results, the following results can be obtained, which are shown in the following Figure 1, 2, 3 , 4. It can be seen from the following chart that the prediction results of the interval settlement of the BP neural network and the wavelet neural network are compared with the actual settlement results. As the number of training increases, the error gradually increases, and the cumulative settlement prediction results are in good agreement with the actual results. 


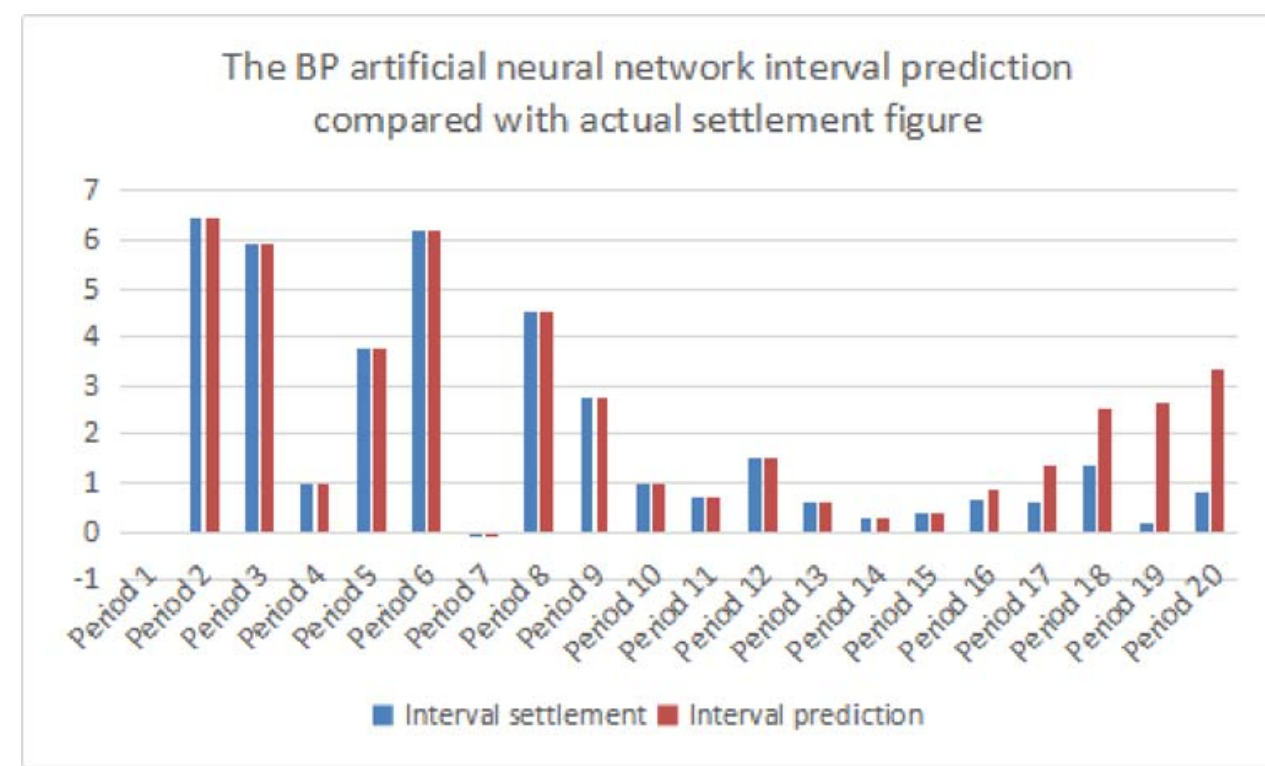

Figure 1 BP Comparison of Artificial Neural Network Interval Prediction and Actual Settlement Accumulation of BP artificial neural network prediction compared with actual settlement figure

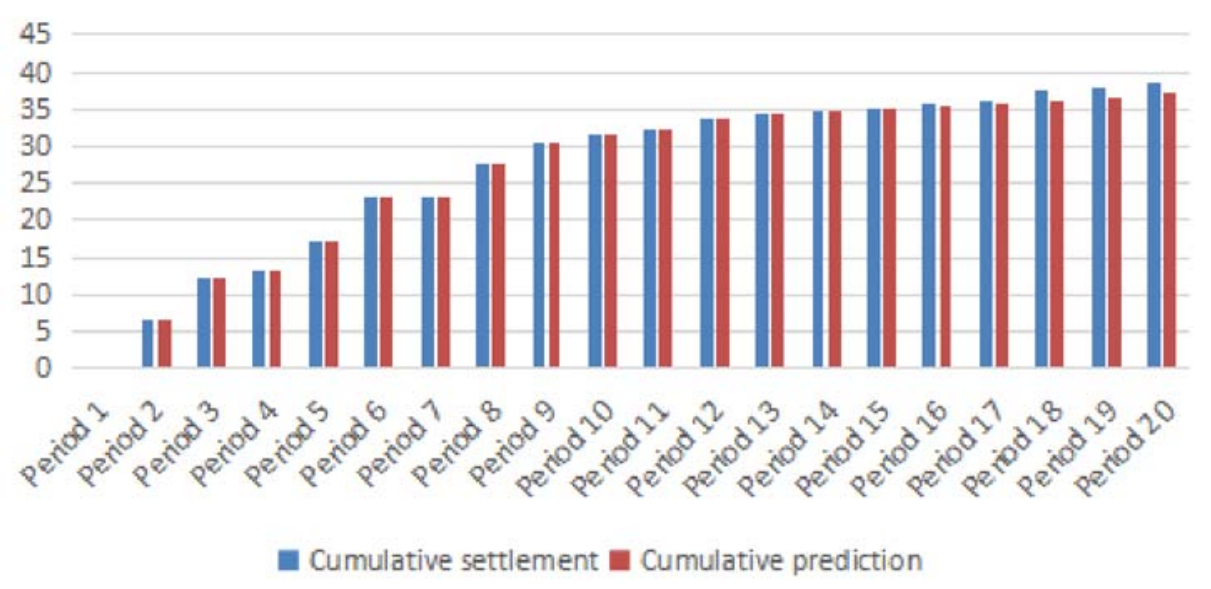

Figure 2 Comparison of BP artificial neural network cumulative prediction and actual settlement Wavelet neural network interval prediction compared with actual settlement figure

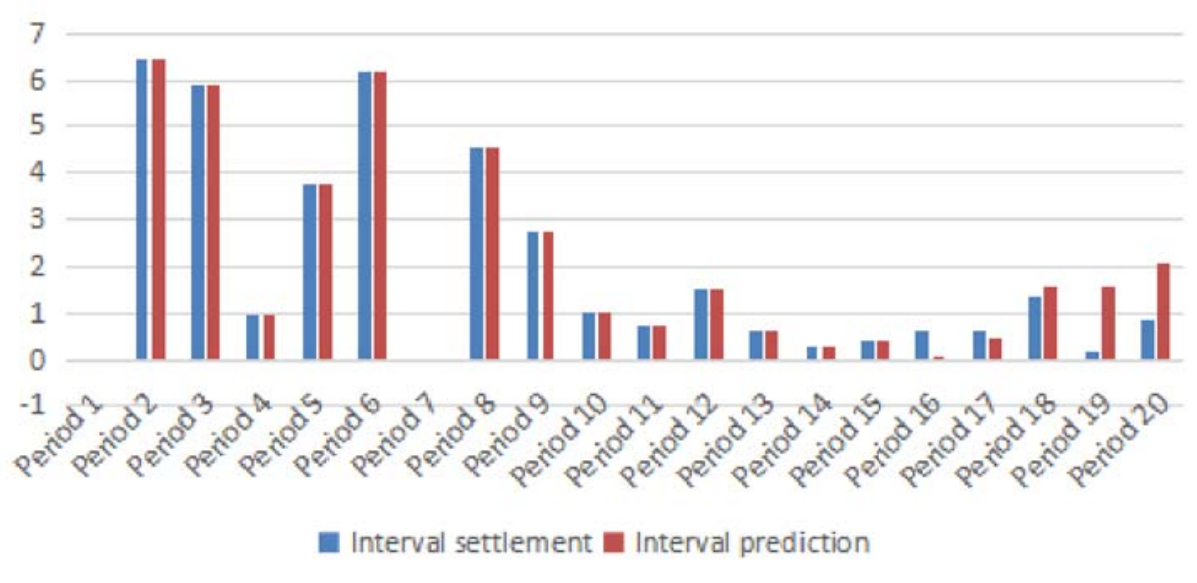

Figure 3 Comparison between wavelet neural network interval prediction and actual settlement 


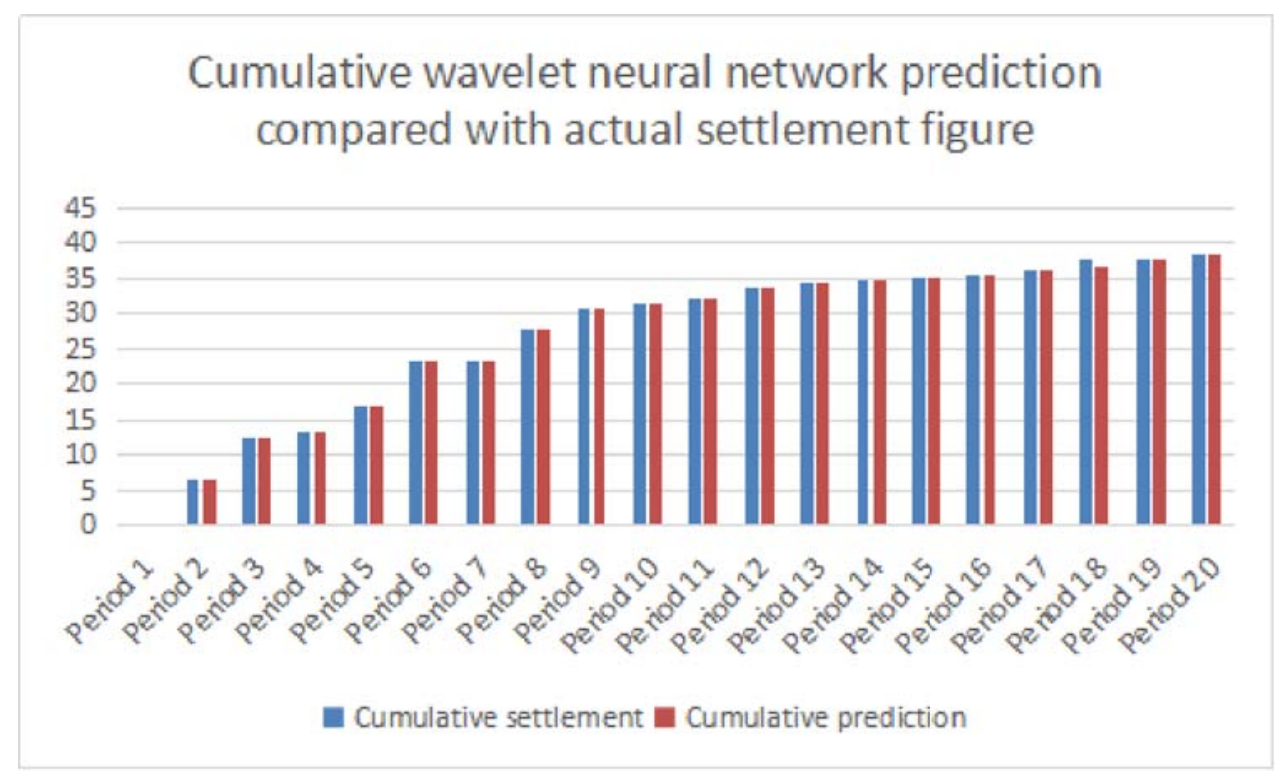

Figure 4 wavelet neural network prediction compared with the actual settlement

\section{THE ACTUAL APPLICATION EFFECT OF WAVELET NEURAL NETWORK}

Using the data from the references to perform operations on the wavelet neural network and the BP neural network, and comparing the calculated results, it is found that the processing effects of the two are very different. The error curve of the training process is shown in Figure 5 and Figure 6.

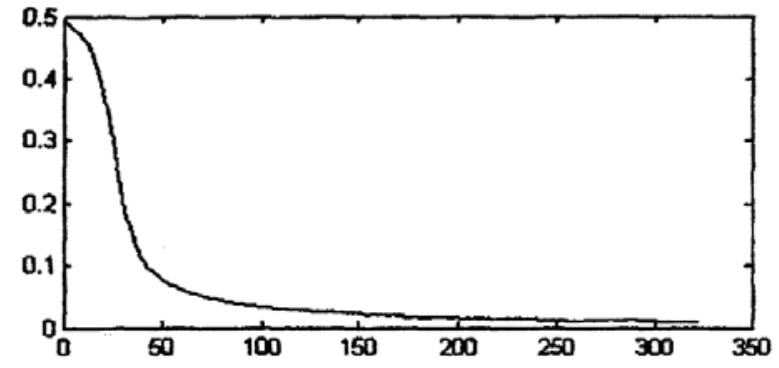

Figure 5 Error curve of wavelet neural network (embedded)

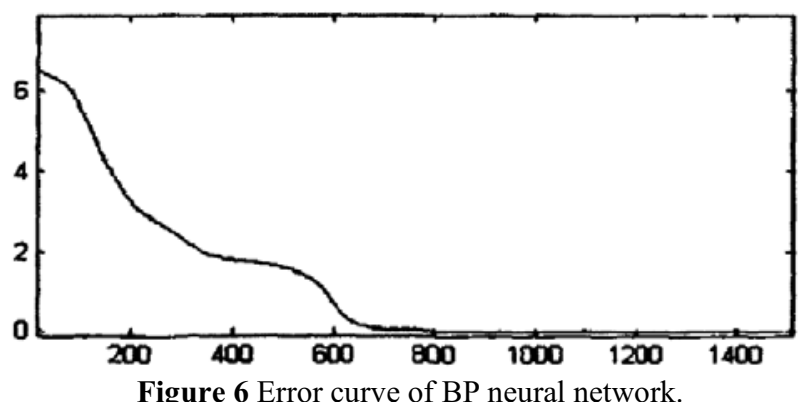

(1) From the perspective of the approximation nature of the function, both wavelet neural network and BP neural network have consistent approximation capabilities.

Due to the fast attenuation of wavelet function, wavelet neural network is a local approximation network, but BP neural network is a global approximation network. The global approximation network uses a global activation function, so neurons can be activated within a large input range, and all activation functions overlap with each other, thereby generating cross-effects. During the training process, all interacting neurons must be connected. The weights are adjusted to reduce the learning speed of global approximation. Because the optimization problem is non-linear, the BP neural network cannot guarantee the convergence of its learning and may fall into a local minimum. In addition, the global approximation network has outputs in the entire input range, regardless of the density of the training data in the input space, which will cause a large error.

By using the local activation function, we can get the local approximation network and overcome the above disadvantages of the global approximation network. For each input, only a few neurons are activated. Because only part of the network needs to be adjusted. These nerves can easily adapt to the new data. Also the local characteristics of the activation function ensure the participation of a few activated neurons. Therefore, compared with the global 
network, the local network has the advantages of fast convergence speed and easy to adapt to the new data, which can avoid the large error of combining wavelet analysis with neural network.

(2) From the structural form, there is no essential difference between wavelet neural network and BP neural network. The difference between them is: the hidden node function of the wavelet network is a wavelet function, and the weights and thresholds from the input layer to the hidden layer correspond to Wavelet expansion and translation parameters.

(3) From the point of view of the parameter learning algorithm, since the weight coefficient of the wavelet network has a linear relationship with the output of the network, the weight coefficient can be obtained by using a linear optimization method. Especially for the orthogonal wavelet network, the method of solving the weight value is the only solution; and for the BP neural network, due to the nonlinear relationship between its adjustable parameters and the network output, parameter estimation must be based on nonlinear optimization techniques, resulting in long learning time and slow convergence. When learning by gradient descent, it is easy to fall into the local minimum.

In short, wavelet network is a feed-forward network formed by introducing wavelet analysis theory into neural network. Compared with traditional neural network, it has the following advantages:

(1) The entire structure of the wavelet network is determined based on the wavelet analysis theory, thus avoiding the blindness in the design of the network and other structures.

(2) Certain parameters in the network can be determined with the help of some physical meanings of the signal. For example, the translation and scaling parameters can be determined according to the time-frequency characteristics of the signal.

(3) The network weight learning algorithm is simpler than the conventional BP neural network learning algorithm. Besides, the error function is a convex function with respect to the weight. In addition, there is no local minimum point, the convergence speed is faster, and the accuracy is higher.

\section{CONCLUSIONS}

Regardless of whether it is interval settlement or cumulative settlement, it can be seen from the prediction results that the prediction results of the wavelet neural network are basically better than the prediction results of the BP artificial neural network, and the number of trainings is greatly reduced. In the interval settlement prediction, the training times of BP artificial neural network is 8637 times, while the training times of wavelet neural network is 231 times, a reduction of about $97.32 \%$; in the prediction of cumulative settlement, the number of BP artificial neural network training is 5,864; while the number of wavelet neural network training is 223 , a reduction of about $96.2 \%$. The settlement of this observation site is severe in the early stage and tends to be stable in the later stage. The prediction accuracy of the BP artificial neural network is poor. In this case, the adaptive prediction ability of the wavelet neural network is particularly obvious, and the prediction accuracy is significantly improved. In the interval settlement prediction, it increased by about $39.48 \%$, and in the cumulative settlement prediction, it increased by about $32.43 \%$. From the model accuracy of the above settlement prediction example results, it can be seen that no matter which model is used, interval settlement prediction is better than cumulative settlement in most cases. However, if interval settlement prediction is used for all the cases, although in most cases a better prediction effect can be achieved, there are still some cases where the prediction effect is not as good as the cumulative settlement prediction.

Wavelet analysis has good multi-resolution characteristics and can extract local information of signals, so it is an effective method to remove data noise. Neural networks have good learning and memory, which can store knowledge distributed, have strong fault-tolerant ability, and can approximate any complex nonlinear system, so it is widely used in prediction, classification, recognition and other fields. Moreover, the combination of wavelet analysis and neural network prediction in building settlement monitoring and prediction can obtain higher prediction accuracy and better prediction effect, which is a prediction method with great development potential.

\section{Acknowledgment}

I would like to express my gratitude to all those who helped me during the process of writing of this thesis. My deepest gratitude goes first and foremost to my teachers and professors in my university, who have provided me with valuable guidance in every stage of the writing of this thesis,and for their constant encouragement and guidance. In the preparation of this thesis, they have spent much time reading through each draft and provided me with inspiring advice. Without their instruction,this thesis could not have reached its present form. Further, I would like to thank all my parents and friends for their encouragement and support. Without all their enlightening instruction and impressive kindness, I could not have completed my thesis.

\section{References}

1. Y. Wang. Study on deformation monitoring and prediction of BP neural network based on wavelet denoising. Guilin University of Technology, (2017).

2. Z. Dong. Analysis on the Destructive Effect of Land Subsidence on Urban Sustainable Development. Journal of Ningbo University (Science and Technology Edition), 3:354-356, (2004)

3. L. Zhou, S. Liu. The influence and analysis of ground deformation and settlement on the environment. Coal Technology, 2: 107-108, (2006).

4. X. Lu, H. Liu. Wavelet Analysis Technology in Analytical Chemistry. Chemical Industry Press, (2006).

5. L. Zhang, X. Huang, W. Feng, X. Wang. Application of Artificial Neural Network in Settlement Prediction 
of Embankment. Road Traffic Technology, 5:7-10, (2006).

6. H. Luo. Research on prediction method of settlement time series. Guangxi University, (2005). 